\title{
Wiring the Ear: Instrumentality and Aural Primacy in and After David Tudor's Unstable Circuits
}

The early 20th century saw a spate of innovative electronic musical instruments. For instance, the theremin (1919) and Ondes Martenot (1928) not only offered new sound generation techniques, but married them to similarly innovative means of interaction. However, by the late 1920s, the development of novel performance interfaces had stalled, and the familiar organ-type keyboard re-appeared on many electronic instruments of the 1930s (Manning 2004, pp. 4-6). Moreover, as the era of the tape-based studio began postwar, the link between electronic music and live performance seemed to recede (Ibid., pp. 19-74). Compared to the limited timbres of most earlier electronic instruments, the sound creation and manipulation possibilities of tape were more sophisticated. However, splicing together even a short piece could take months of toil. Thus, by the mid-1960s, a number of real-time alternatives had emerged, from Stockhausen's electronic processing of acoustic instruments, to the modular synthesizer, and the live electronics of David Tudor.

At a time when studio equipment and commercial synthesizers were unavailable to most people, Tudor's 'homebrewed' circuits suggested a more accessible model of electronic music. They also exhibited a number of distinctive characteristics that set them apart from contemporary music technologies and acoustic instruments. Firstly, in contrast to the nonreal-time workflow and fixed outcomes of magnetic tape, the Tudor electronics emphasized liveness and spontaneity. Second, while consistency and repeatability are fundamental to most traditional instruments and commercial synthesizers, the complex feedback paths built into Tudor's circuits courted instability and unpredictability. Thus, rather than precise control, the performer exerted only loose influence over a precariously drifting system, and the same input could produce different results each time. Indeed, at times, Tudor's electronics could produce sound without any input from the performer. Finally, if most traditional instruments are essentially unchanging over time; static targets for performers to grow into and master, Tudor commonly reconfigured his circuits between performances. Many were also sensitive to slight changes in their setup or environment. Thus, radically different musical outcomes and divergent behaviors could transpire from one performance to the next. For Holzer (2011), these characteristics reflect Tudor's particular post-Cagean outlook. That is, a shift from performance as accurate reproduction or expressive deviation, to the openended exploration of a specific set of possibilities delineated by an instrument-composition.

Collectively and cumulatively these properties destabilize established notions of what it means to play and learn a musical instrument. The notion of instrumental mastery is particularly challenged. Instrumental mastery relates to the development, typically over an extended period of time, of the skills required to play a musical instrument. Conventional understandings tend to focus on the technical motor abilities and the physical dexterity required to accurately and consistently produce specific musical outcomes. This physical bias is rooted in the nature of the acoustic performer-instrument relationship. For instance, most experienced musicians do not primarily rely on their eyes and ears to navigate the interface of their instrument (even if these play some part). They instead utilize the subtle haptic feedback that results from the close coupling between performance interface and sound generation 
mechanism (Marshall 2008). This feedback has two functions. Moment-to-moment, it enables the performer to ascertain the state of the instrument and subtly adjust their actions to suit. Additionally, over time, this 'feel' of the instrument as it responds to different input gestures becomes internalized in body memory.

The performer-instrument interactions in Tudor's live electronics are quite different to the acoustic domain. For instance, rather than gestural input being transferred to the means of sound generation by a performance interface, the performer influences the sound generation circuit far more directly. This includes adjusting potentiometers, but also touching exposed parts of the circuit itself. Both result in notably little performance spectacle. Additionally, like many electronic instruments, the sounds produced by the Tudor circuits are diffused externally, by loudspeaker or transducer. Without acoustic vibrations at the point of performer-instrument contact to provide haptic feedback, the performer must instead rely on aural cues to grok the response of the instrument. However, this aural turn in feedback modality significantly blurs the clarity of feedback. For instance, while haptic feedback is localized by the performance interface, the auditory environment is comparatively expansive, loosely bounded and already crowded (by the musical output of the instrument, environmental sounds, etc.). Thus, added to interaction based more on loose influence than tight causality, there are considerable navigational difficulties.

It is clear that these peculiarly Tudorian characteristics require a reformulation of instrumental mastery. In particular, rather than increasingly sophisticated physical manipulation of a consistent acoustic object, mastery must instead pertain to the ephemeral aural and mental skills required to process the acoustic environment and intelligently translate it into abstract gestural response. It must also embrace a kind of learned 'letting go': an acceptance that the instrument's response may never be entirely knowable, and that the instrument itself may change over time in ways hitherto unimagined.

Although Tudor resisted computers in his own work, these ideas are pertinent to the musical use of computers today. For instance, Cascone (2001) remarks that audiences struggle to adapt to the minimal gestural spectacle of laptop music. More relevant still is Jordà's (2005) comment that planned obsolescence and the tendency to endlessly upgrade has resulted in few digital musical instrument virtuosi. Perhaps it is instead our notion of virtuosity that needs to be adjusted; and better understanding Tudor provides one possible basis for this.

\section{REFERENCES}

Cascone, K. (2000) The Aesthetics of Failure: "Post-Digital" Tendencies in Contemporary Computer Music, Computer Music Journal, 24(4): pp. 12-18.

Holzer, D. (2011) Schematic as Score: Uses and Abuses of the (In)Deterministic Possibilities of Sound Technology [online]. Vague Terrain 19: Schematic as Score. Available: http://web.archive.org/web/20150227025105/http://vagueterrain.net/journal19 [Accessed 20 December 2015].

Jordà, S. (2005) Digital Lutherie: Crafting Musical Computers for New Musics' Performance and Improvisation. Ph.D. thesis, Universitat Pompeu Fabra. 
Marshall, M.T. (2008) Physical Interface Design for Digital Musical Instruments. Ph.D. thesis, McGill University.

Manning, P. (2004) Electronic and Computer Music. New York: Oxford University Press. 